\title{
Targeted genomic analysis of cutaneous T cell lymphomas identifies a subset with aggressive clinicopathological features
}

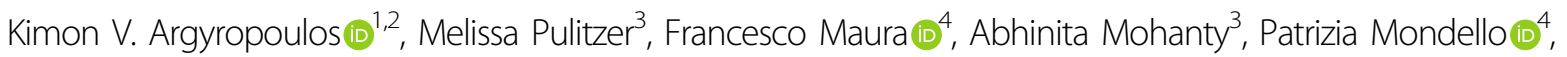 \\ Steven M. Horwitz ${ }^{4}$, Patricia Myskowski $i^{4}$, Alison Moskowitz ${ }^{4}$, Ahmet Dogan $0^{3}$, Christiane Querfeld ${ }^{5}$, Franck Rapaport ${ }^{6}$, \\ Marina Siakantaris ${ }^{2}$, Peter C. Louis ${ }^{7}$, Natasha Galasso ${ }^{4}$, Marcel R. M. van den Brink ${ }^{1,4}$ and M. Lia Palomba (1) ${ }^{4}$
}

\section{Dear Editor,}

Cutaneous T-cell Lymphomas (CTCLs) have been shown to have a complex mutational landscape. Despite the availability of molecular data it is unclear whether they have any diagnostic and prognostic utility. In this study, we performed targeted sequencing for 585 genes that are frequently mutated in solid and hematological malignancies (MSKCC HemePACT) in 77 CTCL samples, including lesions from early Mycosis Fungoides (eMF) $(n=21)$, advanced Mycosis Fungoides/large cell transformation (aMF-LCT) $(n=15)$, Sézary syndrome (SS) $(n$ =17), CD30+ Lymphoproliferative Disorders (CD30 $+\mathrm{LPD})(n=12), \gamma \delta$ CTCLs $(n=5)$ and other rare CTCLs $(n=7)$. We identified genetic alterations in 358 genes with eMF lesions showing the lowest mutational burden, while aMF lesions showed the highest mutational burden among all subtypes. $\mathrm{C}>\mathrm{T}$ transitions were the predominant substitution among all subsets with the exception of eMF lesions. Although there was remarkable pathway heterogeneity, all CTCL histological subsets carried mutations in the GPCR/RTK/MAPK signaling pathway. Only four genes were recurrently mutated in more than $10 \%$ of CTCLs: CDKN2A/B, PCLO, FAT1, and TP53. We identified that the presence of mutations in at least one of those genes defined a CTCL subset with increased tumor burden, aggressive immunopathological features, and dismal prognosis.

\footnotetext{
Correspondence: M. Lia Palomba (Palombam@mskcc.org)

${ }^{1}$ Department of Immunology, Memorial Sloan Kettering Cancer Center York, New York, NY, USA

${ }^{2}$ National and Kapodistrian University of Athens, Athens, Greece

Full list of author information is available at the end of the article

These authors contributed equally: Kimon V. Argyropoulos, Melissa Pulitzer,

Marcel R. M. van den Brink, M. Lia Palomba
}

CTCLs constitute a heterogeneous group of lymphoproliferative neoplasms, which differ widely in terms of biology, histopathology, and clinical presentation ${ }^{1,2}$. Whole-genome, whole-exome, and targeted sequencing approaches have identified a complex mutational landscape, affecting genes involved in immune-synapse signaling, cell-cycle regulation, and epigenetic modulation $^{3-11}$. Most studies thus far have focused on MF and SS, while the molecular features of rarer CTCLs are not fully elucidated and there is a lack of meaningful molecular signatures that could be used in the clinical setting for all CTCLs. The goal of this study was (1) to characterize concomitantly multiple CTCL subsets and identify the predominant genetic events that characterize each entity and (2) to identify clinically meaningful molecular CTCL subgroups.

We performed targeted sequencing for 585 genes frequently mutated in solid and hematological malignancies (MSKCC HemePACT ${ }^{12}$ ) in 77 CTCL samples with paired germline control samples when available $(n=43)$. All patients had previously signed informed consent to have their specimen used for research purposes, in accordance with the Declaration of Helsinki and approval by the Memorial Sloan Kettering Cancer Center Institutional Review Board. Figure 1c and Supplementary Table 1 summarize patient demographics and clinicopathological characteristics. Germline and neoplastic material was sequenced on a HiSeq2500 Illumina instrument. Detailed description of mutation analysis and histological/immunophenotypic analysis can be found in the Supplementary material.

We identified single nucleotide variants (SNVs), copy number alterations (CNAs) and structural variants (SVs) 


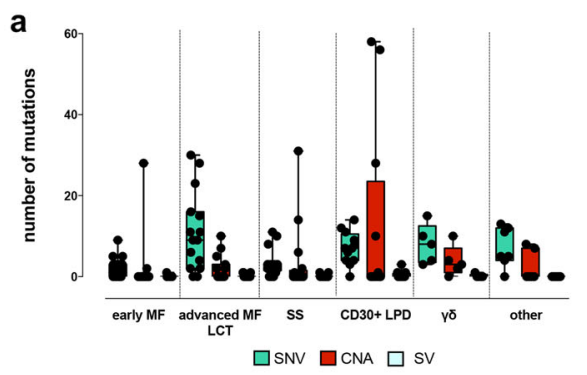

b

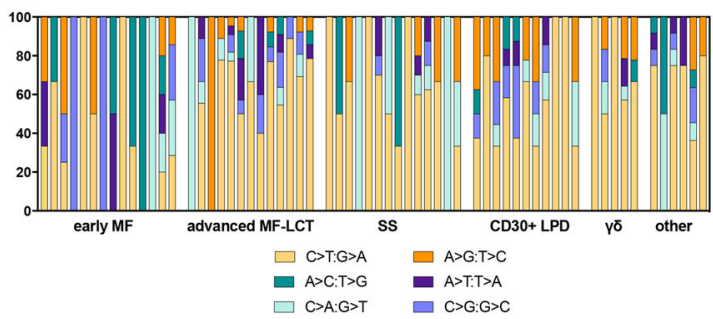

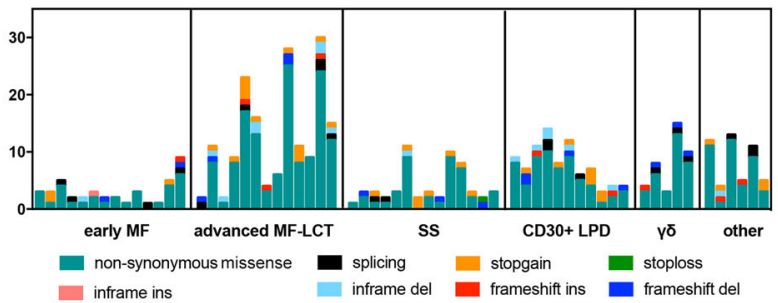
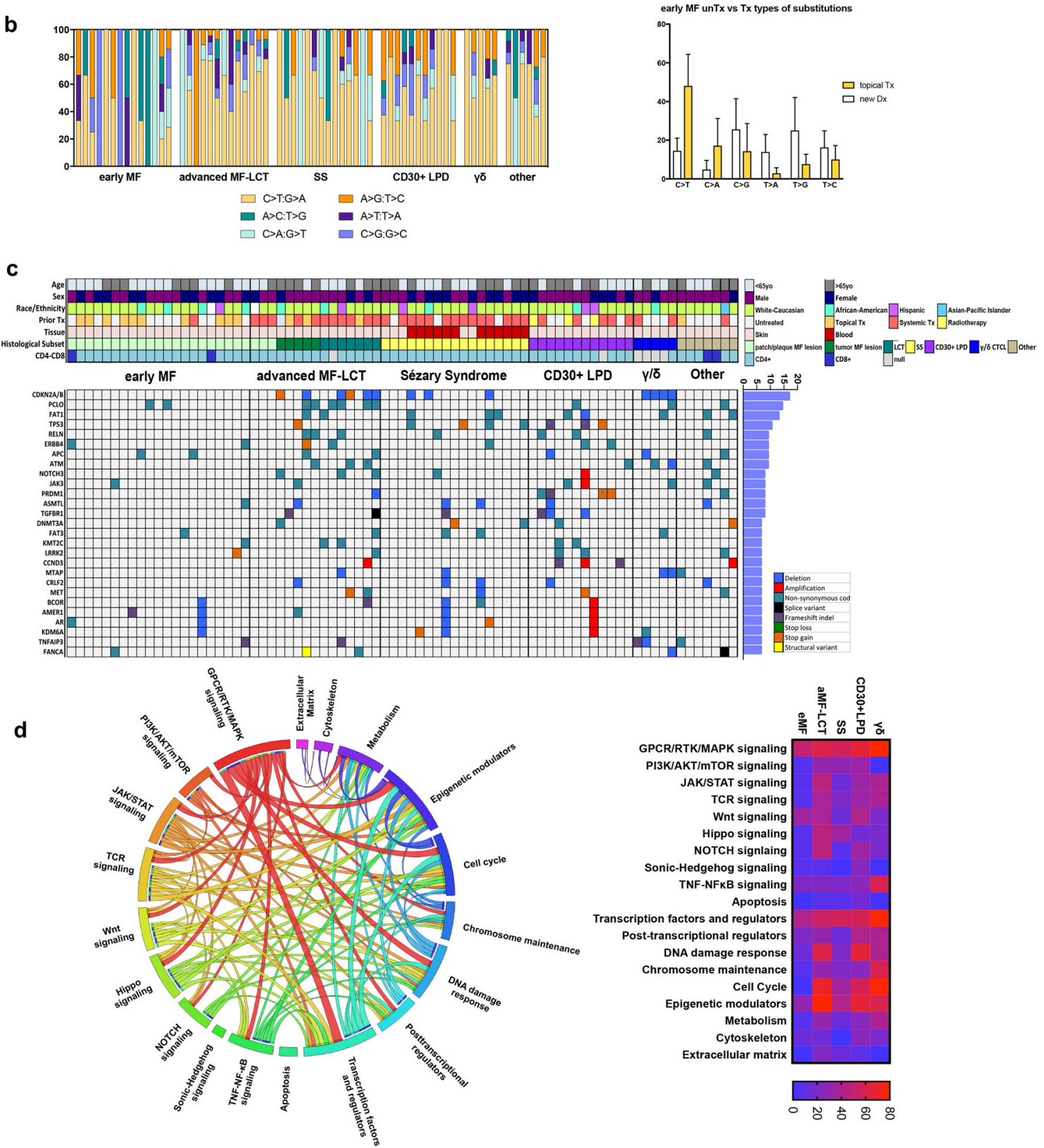

Fig. 1 Genomic alterations and biologic pathways in CTCL. a Illustration of SNV, CNA, and SV load (left) and representation of the identified SNV types (right) in different CTCL subsets. b Base substitutions identified in different CTCL subsets (left) and base substitutions in newly diagnosed/ untreated versus topically treated eMF lesions (right). c Oncoprint of recurrent (>5\%) alterations incorporating SNVs, CNAs, and SVs. The first seven rows represent corresponding demographic and clinicopathologic data. d CIRCOS plot illustrating 19 recurrently mutated pathways and their cooccurence in CTCLs (left), as well as the frequency of mutated pathways in different CTCL subsets (right). 
in 358 genes, with $537 \mathrm{X}$ sequencing depth being the median for all filtered somatic variants (Supplementary Table 2). Early MF (eMF) lesions showed a low mutational burden, with $33.3 \%$ of cases having no detectable alterations (Fig. 1a). Advanced MF and large cell transformed (aMF-LCT) lesions showed the highest mutational burden among all subtypes. Interestingly, 4 out of $12 \mathrm{CD} 30+$ lymphoproliferative disease (CD30+ LPD) samples exhibited multi-chromosomal CNAs. No difference in mutational burden or type of substitution was observed when data were analyzed according to sex, age, or race/ ethnicity (Supplementary Fig. 1a, b, c). C>T transitions were the most prominent events among all CTCLs (Fig. 1b). In eMF lesions, we observed a bias towards $C>T$ transitions primarily in samples that had received prior topical treatment rather than newly diagnosed/treatment naïve samples. This was not related to an inherent aggressiveness of the treated lesions, as the two eMF groups had a similar distribution of stage IA and IB cases (Supplementary Fig. 1d) and no difference in variant allele frequency (VAF) numbers, which reflect the tumor content of the lesion (Supplementary Fig. 1e, f). Further mutational signature analysis showed that the single base substitution (SBS) signature related to ultraviolet (UV) damage represented $34.8 \%$ of events (Supplementary Fig. $2 \mathrm{a}, \mathrm{b})$. Although $44.1 \%$ of our specimen were from patients that had received systemic therapy, no chemotherapy-related SBS signatures were identified.

Forty-six genes showed recurrent alterations in more than 5\% of CTCLs (Fig. 1c). Regardless of CTCL histology, alterations in more than $10 \%$ of the cohort were identified in CDKN2A and B, PCLO, FAT1, and TP53 (Supplementary Fig. 3). CDKN2A and B alterations occurred in $16.9 \%$ of CTCL $(n=13$, SNVs, $n=2$; gene deletions, $n=11)$. PCLO exhibited the highest frequency of SNVs (14.9\%, $n=11$ sample, $n=15$ mutations), which were all missense mutations, with one sample carrying an additional stop codon gain mutation. FAT1 harbored somatic alterations in $13 \%$ of CTCL $(n=10$, SNVs, $n=9$; gene deletions, $n=1$ ), while its homolog FAT3 showed missense SNV in $6.5 \%$ of CTCL $(n=5)$. TP53 was mutated in $10.4 \%(n=8)$ of all samples, harboring missense variants $(n=3)$, stop gain variants $(n=3)$, and frameshift deletions $(n=2)$. Other recurrently mutated genes (frequency $>7 \%$ ) included RELN, ERBB4, APC, ATM, NOTCH3, JAK3, PRDM1, ASMTL, and TGFBR1. Despite the heterogeneity of the genomic landscape in CTCL, and although no recurrently mutated gene was specific for a CTCL subtype, we observed differential mutated gene distribution between CTCL subtypes. CDKN2A or B alterations were present in aMF-LCT, SS, and $\gamma \delta C T C L$ samples $(40 \%, 17.6 \%$, and $80 \%$, respectively), and absent from eMF, CD30+LPD, and other rare CTCL subsets. Moreover, although PCLO was mutated in $40 \%$ of
aMF-LCT, it was not mutated in SS, while the majority of TP53 mutations occurred in SS and CD30+LPD (23.5\% and $25 \%$, respectively). CD30+LPD showed recurrent (25\%) alterations in TGFBR1, PRDM1, CCND3, PTCH1, and POLE. Finally, $\gamma \delta C T C L$ showed recurrent $(40 \%)$ alterations in ATM, MTAP, TNFAIP3, SOCS1, and SMC3. Pathway analysis identified at least 19 distinct pathways involved in CTCLs with (a) GPCR/RTK/MAPK signaling molecules, (b) transcription factors and regulators, and (c) epigenetic modulators being mutated in more than $50 \%$ of the samples. GPCR/RTK/MAPK signaling was within the top three mutated pathways involved in all CTCL histological subtypes. Cell cyclerelated alterations never occurred in eMF patches or plaques, yet they were highly prevalent in tumor MF or LCT lesions, suggesting that the acquisition of such events might contribute to tumor progression or LCF of eMF lesions (Fig. 1d).

Since eMF was mutationally silent and carried a significantly more indolent course compared to all other CTCL subsets in a retrospective analysis of overall survival (Fig. 2a), we focused on identifying molecular events that can discriminate noneMF CTCLs with aggressive histopatholgical and clinical features, which are indistinguishable under the current WHO classification system. Due to the high number of nonrecurrent alterations, unsupervised clustering analysis failed to classify samples into large enough groups that could be further analyzed for clinicopathological correlations (Supplementary Fig. 4). We then classified non-eMF CTCLs based on the absence (Signature A, $n=28$ ) or presence (Signature B, $n=28$ ) of mutations in at least one of the four most frequently altered genes: CDKN2A/B, PCLO, FAT1, and TP53. CTCL subsets did not show a differential distribution between the two groups (Fig. 2b). Signature B samples had a significantly higher mutational burden, suggesting that this signature could be a surrogate for hypermutated CTCLs (Fig. 2c). From a morphological standpoint signature B samples had a significantly higher presence of epiderrmotropism and Pautrier microabscess formation, while they exhibited a significantly higher incidence of karryorhexis, which is an indicator of higher cell-turnover rate (Fig. 2d). In regard to $\mathrm{T}$ cell polarization, despite the heterogeneity in expression and varying coexpression levels of Tbet, GATA3, FoxP3, and Bcl6, malignant $\mathrm{T}$ cells showed a Th2-predominant phenotype, as it has been previously described (Supplementary Fig. 5a, b) ${ }^{13,14}$. When broken down by molecular subtype, signature B samples exhibited significantly higher GATA3 and lower Tbet expression (Fig. 2e). No difference was observed in FoxP3 and Bcl6 expressions (data not shown). Finally, overall survival univariate analysis in non-eMF CTCLs showed a significantly more aggressive disease course in patients carrying signature $\mathrm{B}$ (Fig. 2f). Multivariate analysis including histological types, age, and prior treatment was also preformed and showed significantly worse outcomes in patients with signature B 


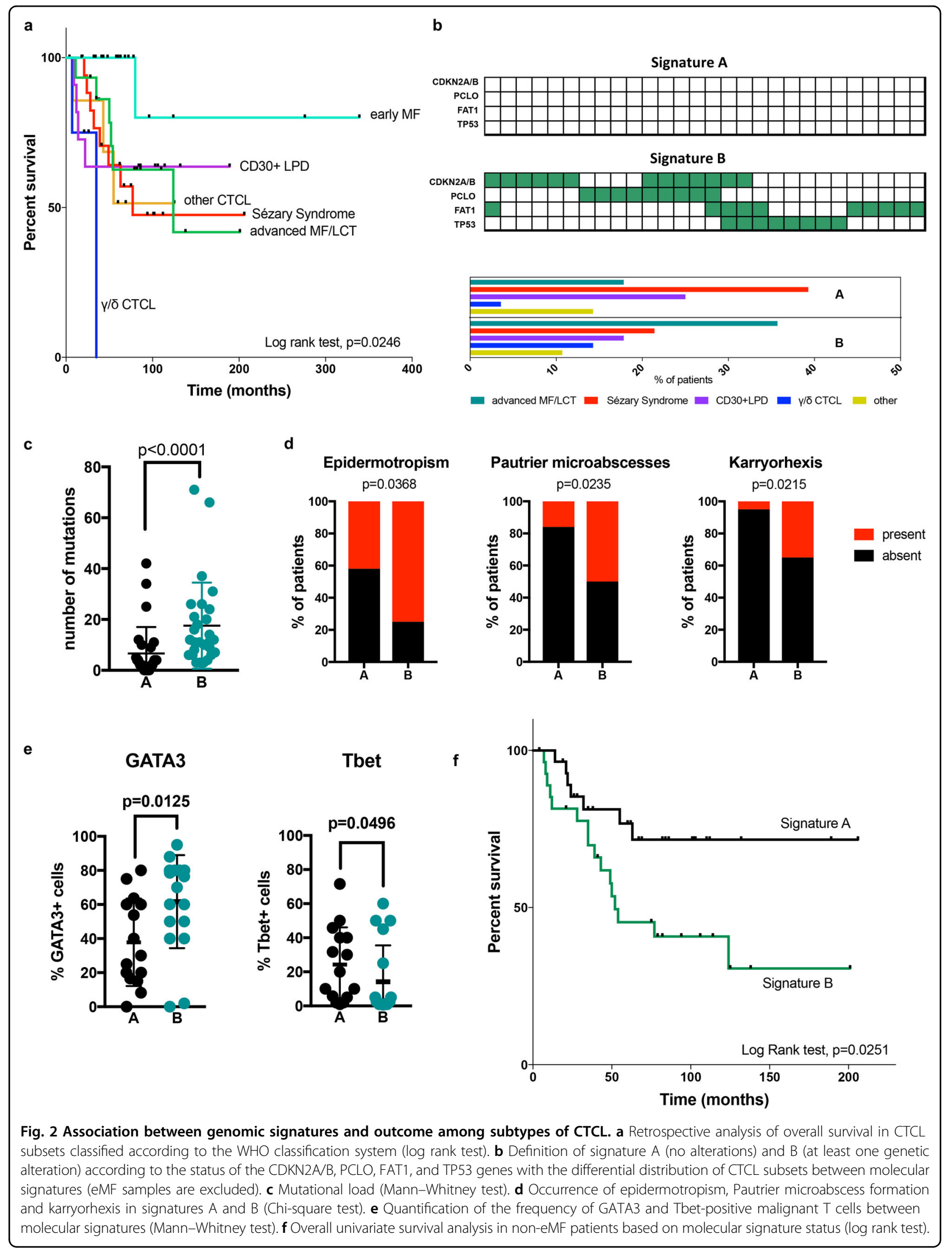


(Supplementary Fig. 6). This is in par with our immunopathological data, considering the established negative prognostic significance of GATA3 positivity in mature $\mathrm{T}$ cell lymphomas ${ }^{15}$. It is worth noting that survival analysis based on the mutation status of for each single gene showed no statistically significant difference compared to WT individuals, with the exception of CDKN2A/B, which only showed a trend for worse outcomes in mutated individuals (Supplementary Fig. 7). Therefore, the concomitant assessment of all four frequently mutated genes appears to be a powerful tool for the prognostication of non-eMF CTCLs, which cannot be provided by the current clinicopathological WHO classification system. Whether all four genes represent true disease drivers and there is biological synergy between them remains to be answered. In summary, CTCLs show few recurrent mutations, which highly overlap between different histological subsets. This study shows that the examination of the mutational status of CDKN2A/B, FAT1, PCLO, and TP53 can be used as a surrogate marker for hypermutated CTCLs with aggressive pathological features and poor prognosis.

\section{Acknowledgements}

We would like to thank Dr. Katya Manova and the personnel of the Molecular Cytology Core Facility at Memorial Sloan Kettering Cancer Center, as well as the Pathology Core Facility for the help with immunohistochemistry studies. This work was supported by The Lymphoma Foundation, the Greenberg Lymphoma Research Award (MSKCC), and the P30 CA008748 MSK Cancer Center Support Grant/Core Grant.

\section{Author details}

'Department of Immunology, Memorial Sloan Kettering Cancer Center York, New York, NY, USA. ${ }^{2}$ National and Kapodistrian University of Athens, Athens, Greece. ${ }^{3}$ Department of Pathology, Memorial Sloan Kettering Cancer Center York, New York, NY, USA. ${ }^{4}$ Department of Medicine, Memorial Sloan Kettering Cancer Center York, New York, NY, USA. ${ }^{5}$ City of Hope Hospital, Duarte, CA, USA. ${ }^{6}$ Center for Hematologic Malignancies, Memorial Sloan Kettering Cancer Center York, New York, NY, USA. 'Department of Biomedical Informatics, Vanderbilt University Medical Center, Nashville, TN, USA

\section{Author contributions}

K.V.A., M.P., M.R.M.V.B., and M.L.P. conceived and designed the study. S.M.H., P. M., A.M., A.D., C.Q., and N.G. helped with the collection of clinical samples. K.V.A. performed experiments. Bioinformatic analysis was performed by A.M., F.R., and F.M., K.V.A. performed mutation calling and data illustration. K.V.A. and M.P. evaluated H\&E morphology and analyzed immunohistochemistry data. K.V.A. and P.M. performed flow cytometry analysis and statistical analysis. K.V.A. wrote the manuscript. M.L.P., M.S., and M.P. reviewed and edited the manuscript.

\section{Data availability}

The data can be made available upon request.

\section{Conflict of interest}

S.M.H. is a consultant for Astex, Celgene, Affimed, Infinity/Nerastem, Millennium/Takeda and Merck. Research grants include Corvus, Celgene, Infinity/Nerastem, Millennium/Takeda, Seattle Genetics, Forty-Seven, Aileron, ADCT Therapeutics, Trillium, Daichii, Portola. A.D. has received personal fees from Roche, Corvus Pharmaceuticals, Physicians' Education Resource, Seattle Genetics, Peerview Institute, Oncology Specialty Group, Pharmacyclics, Celgene, Novartis, Takeda and research grants from National Cancer Institute and Roche. A.M. has received research support from Seattle Genetics, Merck,
Bristol-Myers Squibb, Incyte. She has received honorarium from Kyowa Hakko Kirin Pharma, Miragen Therapeutics, Takeda Pharmaceuticals, ADC

Therapeutics, Seattle Genetics, Cell Medica, Bristol-Myers Squibb, Erytech Pharma. C.Q. is a Steering Committee member of Miragen, is in the Advisory Board of Helsinn/Actelion, Bioniz, Trillium, Kyowa Kirin, Medivir and Mallinckrodt; and receives research funding from Celgene. M.R.M.v.B. has received research support from Seres Therapeutics; has consulted, received honorarium from or participated in advisory boards for Seres Therapeutics, Flagship Ventures, Novartis, Evelo, Jazz Pharmaceuticals, Therakos, Amgen, Magenta Therapeutics, Merck \& Co, Inc., Acute Leukemia Forum (ALF), and DKMS Medical Council (Board); has IP Licensing with Seres Therapeutics, Juno Therapeutics, and stock options from Smart Immune. M.L.P. is a consultant for Merck and Pharmacyclics.

\section{Publisher's note}

Springer Nature remains neutral with regard to jurisdictional claims in published maps and institutional affiliations.

Supplementary Information accompanies this paper at (https://doi.org/ 10.1038/s41408-020-00380-5).

Received: 5 January 2020 Revised: 22 July 2020 Accepted: 17 September 2020

Published online: 09 November 2020

\section{References}

1. Swerdlow S. H. et al. WHO Classification of Tumours of Haematopoietic and Lymphoid Tissues revised 4th edn, 385-402 (International Agency for Research on Cancer (IARC), 2017).

2. Jawed, S. I., Myskowski, P. L., Horwitz, S., Moskowitz, A. \& Querfeld, C. Primary cutaneous T-cell lymphoma (mycosis fungoides and Sezary syndrome): part I. Diagnosis: clinical and histopathologic features and new molecular and biologic markers. J. Am. Acad. Dermatol. 70, 205 (2014). e201-216; quiz 221-202.

3. Vaque, J. P. et al. PLCG1 mutations in cutaneous T-cell lymphomas. Blood 123, 2034-2043 (2014).

4. Choi, J. et al. Genomic landscape of cutaneous T cell lymphoma. Nat. Genet. 47, 1011-1019 (2015).

5. Kiel, M. J. et al. Genomic analyses reveal recurrent mutations in epigenetic modifiers and the JAK-STAT pathway in Sezary syndrome. Nat. Commun. 6, 8470 (2015).

6. Park, J. et al. Genomic analysis of 220 CTCLs identifies a novel recurrent gainof-function alteration in RLTPR (p.Q575E). Blood 130, 1430-1440 (2017).

7. da Silva Almeida, A. C. et al. The mutational landscape of cutaneous T cell lymphoma and Sezary syndrome. Nat. Genet. 47, 1465-1470 (2015).

8. Ungewickell, A. et al. Genomic analysis of mycosis fungoides and Sezary syndrome identifies recurrent alterations in TNFR2. Nat. Genet. 47, 1056-1060 (2015).

9. Fanok, M. H. et al. Role of dysregulated cytokine signaling and bacterial triggers in the pathogenesis of cutaneous T-cell lymphoma. J. Investig. Dermatol. 138, 1116-1125 (2018).

10. McGirt, L. Y. et al. Whole-genome sequencing reveals oncogenic mutations in mycosis fungoides. Blood 126, 508-519 (2015).

11. Wang, L. et al. Genomic profiling of Sezary syndrome identifies alterations of key T cell signaling and differentiation genes. Nat. Genet. 47, 1426-1434 (2015).

12. Durham, B. H. et al. Genomic analysis of hairy cell leukemia identifies novel recurrent genetic alterations. Blood 130, 1644-1648 (2017).

13. Guenova, E. et al. $\mathrm{TH} 2$ cytokines from malignant cells suppress $\mathrm{TH} 1$ responses and enforce a global TH2 bias in leukemic cutaneous T-cell lymphoma. Clin. Cancer Res. 19, 3755-3763 (2013).

14. Nakajima, R. et al. Interleukin-25 is involved in cutaneous T-cell lymphoma progression by establishing a $\mathrm{T}$ helper 2-dominant microenvironment. Br. J. Dermatol. 178, 1373-1382 (2018).

15. Wang, T. et al. GATA-3 expression identifies a high-risk subset of PTCL, NOS with distinct molecular and clinical features. Blood 123, 3007-3015 (2014). 